\title{
The Metabolic Pattern of Idiopathic REM Sleep Behavior Disorder Reflects Early-Stage Parkinson Disease
}

\author{
Sanne K. Meles ${ }^{1}$, Remco J. Renken ${ }^{2}$, Annette Janzen ${ }^{3}$, David Vadasz ${ }^{3}$, Marco Pagani ${ }^{4-6}$, Dario Arnaldi ${ }^{7}$, Silvia Morbelli ${ }^{8}$, \\ Flavio Nobili ${ }^{7}$, Geert Mayer ${ }^{3,9}$, Klaus L. Leenders*1, and Wolfgang H. Oertel*3,10, and the REMPET Study Group \\ Elisabeth Sittig-Wiegand, Candan Depboylu, Kathrin Reetz' ${ }^{1}$, Sebastiaan Overeem, Angelique Pijpers, Fransje E. Reesink, \\ Teus van Laar, Laura K. Teune, Helmut Höffken, Marcus Luster, Lars Timmermann, Karl Kesper, Sofie M. Adriaanse, Jan \\ Booij, Gianmario Sambuceti, Nicola Girtler, and Cathrine Jonsson
}

\begin{abstract}
${ }^{I}$ Department of Neurology, University of Groningen, University Medical Center Groningen, Groningen, The Netherlands; ${ }^{2}$ Neuroimaging Center, Department of Neuroscience, University of Groningen, Groningen, The Netherlands; ${ }^{3}$ Department of Neurology, Philipps-Universität Marburg, Marburg, Germany; ${ }^{4}$ Institutes of Cognitive Sciences and Technologies, CNR, Rome, Italy; ${ }^{5}$ Department of Nuclear Medicine, Karolinska Hospital, Stockholm, Sweden; ${ }^{6}$ Department of Nuclear Medicine, University of Groningen, University Medical Center Groningen, Groningen, The Netherlands; ${ }^{7}$ Clinical Neurology, Department of Neuroscience (DINOGMI), University of Genoa and IRCCS AOU San Martino-IST, Genoa, Italy; ${ }^{8}$ Nuclear Medicine, Department of Health Sciences (DISSAL), University of Genoa and IRCCS AOU San Martino-IST, Genoa, Italy; ${ }^{9}$ Hephata Klinik, Schwalmstadt, Germany; and ${ }^{10}$ Institute for Neurogenomics, Helmholtz Center for Health and Environment, Munich, Germany
\end{abstract}

Idiopathic REM sleep behavior disorder (iRBD) is considered a prodromal stage of Parkinson disease (PD) and other Lewy body disorders. Spatial covariance analysis of ${ }^{18} \mathrm{~F}-\mathrm{FDG}$ PET data has disclosed a specific brain pattern of altered glucose metabolism in $\mathrm{PD}$. In this study, we identify the metabolic pattern underlying iRBD and compare it with the known PD pattern. To understand the relevance of the iRBD pattern to disease progression, we studied the expression of the iRBD pattern in de novo PD patients. Methods: The iRBD-related pattern was identified in ${ }^{18} \mathrm{~F}-\mathrm{FDG}$ PET scans of 21 patients with polysomnographically confirmed iRBD and 19 controls using spatial covariance analysis. Expression of the iRBD-related pattern was subsequently computed in ${ }^{18} \mathrm{~F}-\mathrm{FDG}$ PET scans of 44 controls and 38 de novo, treatment-naïve PD patients. Of these 38 PD patients, 24 had probable REM sleep behavior disorder (RBD) according to the Mayo Sleep Questionnaire. Neuropsychologic evaluation showed mild cognitive impairment in 20 PD patients (PD-MCl), of whom 16 also had concomitant RBD and roughly half $(11 / 20)$ had bilateral motor symptoms. Results: The iRBD-related pattern was characterized by relative hypermetabolism in the cerebellum, brain stem, thalamus, sensorimotor cortex, and hippocampus, and by relative hypometabolism in the middle cingulate, temporal, occipital, and parietal cortices. This topography partially overlapped with the PD-related pattern (PDRP). The iRBDrelated pattern was significantly expressed in PD patients compared with controls $(P<0.0001)$. iRBD-related pattern expression was not significantly different between PD patients with and without probable RBD, or between PD patients with unilateral or bilateral parkinsonism. iRBD-related pattern (iRBDRP) expression was higher in $\mathrm{PD}-\mathrm{MCl}$ patients than in $\mathrm{PD}$ patients with preserved cognition $(P=0.001)$. Subject scores on the iRBD-related pattern were highly correlated to subject scores on the PDRP $(r=0.94$,

Received Sep. 14, 2017; revision accepted Jan. 17, 2018.

For correspondence or reprints contact: Sanne K. Meles, Department of Neurology, University Medical Center Groningen, Hanzeplein 1, P.O. Box 30. 001, 9700 RB Groningen, The Netherlands.

E-mail: s.k.meles@umcg.nl

${ }^{*}$ Contributed equally to this work.

Published online Feb. 23, 2018.

COPYRIGHT (c) 2018 by the Society of Nuclear Medicine and Molecular Imaging.
$P<0.0001)$. Conclusion: Our results show that the iRBDRP is an early manifestation of the PDRP. Expression of both PDRP and iRBDRP was higher in patients with a more severe form of PD (PD-MCl), which indicates that expression of the 2 patterns increases with disease severity.

Key Words: Neurology; PET/CT; statistical; analysis; ${ }^{18}$ F-FDG-PET; idiopathic REM; sleep behavior disorder; Parkinson's disease related pattern; a-synucleinopathy; metabolic pattern

J Nucl Med 2018; 59:1437-1444

DOI: 10.2967/jnumed.117.202242

$\mathbf{M}$ ost patients with idiopathic REM sleep behavior disorder (iRBD) will develop Parkinson disease (PD) or dementia with Lewy bodies (DLB) on long-term clinical follow-up (1-6). In such patients, REM sleep behavior disorder (RBD) indicates the presence of $\alpha$-synuclein pathology in specific brain stem nuclei that regulate REM sleep (7). It is postulated that over time, the pathologic process spreads to other brain areas (8). When the substantia nigra is reached, the ensuing degeneration of the presynaptic dopaminergic system causes the typical motor features of the disease, at which point a PD diagnosis can be made $(9,10)$. Patients with iRBD, by definition, have not yet developed motor symptoms, and provide a unique opportunity to study the early (prodromal) stages of a patient subgroup with $\alpha$-synucleinopathy (9).

The clinical manifestations of PD are caused by functional changes in multiple neuronal networks, reflected by a typical pattern of abnormal glucose utilization in specific brain regions on ${ }^{18}$ F-FDG PET, referred to as the PD-related pattern (PDRP). The PDRP is characterized by relatively increased metabolism in the thalamus, globus pallidus/putamen, cerebellum, and pons and by relative hypometabolism in the occipital, temporal, parietal and frontal cortices. The PDRP has been consistently identified in several PD populations using spatial covariance analysis (i.e., with 
the Scaled Subprofile Model and Principal Component Analysis [SSM/PCA]) (11-18). Expression of the PDRP can be quantified in new ${ }^{18}$ F-FDG PET scans (12), which can be used to investigate group differences and relationships with clinical characteristics.

PDRP expression was significantly higher in ${ }^{18} \mathrm{~F}$-FDG PET scans of patients with iRBD, than in age-matched controls (1921). Moreover, high baseline PDRP subject scores were associated with a higher risk of developing PD in the next $5 \mathrm{y}$ (19).

$\mathrm{Wu}$ et al. investigated the metabolic topography of iRBD, applying SSM/PCA to the ${ }^{18}$ F-FDG PET data of 21 patients with iRBD and 21 age-matched controls (20). The iRBD-related pattern (iRBDRP) showed partial overlap with the PDRP. Interestingly, iRBDRP expression was high in patients with iRBD and in earlystage PD patients with unilateral parkinsonism (Hoehn and Yahr [H\&Y] stage 1), but lower in more advanced PD patients (H\&Y stage 2), suggesting that the iRBDRP contains altered metabolism in regions specific to the prodromal or early stages of PD.

PD patients with concomitant RBD are thought to have a rapidly progressive subtype of the disease with a higher risk of subsequent cognitive decline (22), underscoring the potential of the iRBDRP to provide insights into the evolution of functional changes in PD from its early stages. In this study, we provide a further identification of the iRBDRP in an independent ${ }^{18} \mathrm{~F}$-FDG PET dataset of $21 \mathrm{iRBD}$ patients and 19 controls. In addition, we study the relationship between the iRBDRP and disease severity by calculating expression of the newly identified iRBDRP in 38 carefully characterized, de novo, treatment-naïve PD patients. We compare iRBDRP expression not only between H\&Y stages 1 and 2, but also between PD patients with and without probable RBD, and between PD patients with mild cognitive impairment (PD-MCI) and those with normal cognition (PD-NC).

\section{MATERIALS AND METHODS}

\section{Participants}

Twenty-one patients with iRBD and 19 age-matched controls (cohort A) were used for identification of the iRBDRP. Cohort B, consisting of
9 patients with iRBD and 13 age-matched controls, was used for validation. Clinical data of both cohorts are provided in Table 1. iRBD was confirmed by video-assisted polysomnography (21). Subjects in cohorts $\mathrm{A}$ and $\mathrm{B}$ underwent ${ }^{18} \mathrm{~F}-\mathrm{FDG}$ PET on a Biograph mCT-64 PET/CT camera (Siemens) as described previously (21).

From a previous study, we included ${ }^{18}$ F-FDG PET data from 44 healthy controls and 38 consecutive outpatients with de novo, drugnaïve PD (Table 2) (23). The Mayo Sleep Questionnaire (24) and a clinical interview by a sleep medicine expert were conducted on each patient. A diagnosis of "probable RBD" was made in 24 patients (PD$\mathrm{RBD}+$ ). The remaining $14 \mathrm{PD}$ patients had no signs or symptoms of RBD (PD-RBD-). On the basis of neuropsychologic assessment, 20 PD patients were diagnosed with MCI, and 18 had normal cognition. Furthermore, 23 PD patients had unilateral motor symptoms (H\&Y stage 1), and 15 had bilateral symptoms (H\&Y stage 2). Disease duration was defined by the number of months patients had motor symptoms before the diagnosis.

The study was approved by the local Institutional Review Boards. Voluntary written informed consent was obtained from each subject after verbal and written explanation of the study, in accordance with the Declaration of Helsinki.

\section{${ }^{18}$ F-FDG PET Data Preprocessing}

All images were spatially normalized onto an ${ }^{18} \mathrm{~F}$-FDG PET template in Montreal Neurologic Institute brain space (25) using SPM12 software (Wellcome Department of Imaging Neuroscience, Institute of Neurology), implemented in MATLAB (version 2012b; MathWorks).

\section{iRBDRP Identification in Cohort A}

The iRBDRP was identified by applying SSM/PCA to the ${ }^{18} \mathrm{~F}-\mathrm{FDG}$ PET data of cohort A. In brief, after anatomic registration, images were masked to remove out-of-brain voxels and log-transformed, and subject and group means were removed, resulting in a residual profile for each scan. PCA was applied to these residual profiles in voxel space, and the components explaining the top $50 \%$ of the total variance were selected for further analysis. For each subject, a score was calculated on each selected principal component (PC). These scores were entered into a forward stepwise logistic regression analysis. The

TABLE 1

Demographic Data of Controls and iRBD Patients (Cohorts A and B)

\begin{tabular}{|c|c|c|c|c|c|c|}
\hline \multirow[b]{2}{*}{ Variable } & \multicolumn{3}{|c|}{ iRDBRP identification (cohort A) } & \multicolumn{3}{|c|}{ iRBDRP validation (cohort B) } \\
\hline & Controls & iRBD & $P^{*}$ & Controls & iRBD & $P^{\star}$ \\
\hline$n$ & 19 & 21 & & 13 & 9 & \\
\hline Age $(y)^{\dagger}$ & $62.4 \pm 7.5(43-70)$ & $61.9 \pm 5.4(50-70)$ & 0.82 & $61.3 \pm 8.6(52-78)$ & $64.2 \pm 6.3(56-78)$ & 0.40 \\
\hline Sex (male/female) & $9 / 10$ & $18 / 3$ & 0.010 & $9 / 4$ & $8 / 1$ & 0.36 \\
\hline MoCA & $29(27-30)$ & $27(25.5-28)$ & 0.003 & $30(28.5-30)^{\ddagger}$ & $28(27-29)$ & \\
\hline UPDRS-III & $0(0-1)$ & $2(1-4)$ & 0.002 & NA & $2(0-2)$ & \\
\hline Age at onset $\mathrm{RBD}^{\dagger}$ & & $55.0 \pm 7.1(37-67)$ & & & $60.8 \pm 6.0(52-73)$ & \\
\hline iRBD duration (y) & & $6(3.5-8.0)$ & & & $4(1.0-5.0)$ & \\
\hline iRBDRP $z$ scores & $0 \pm 1$ & $1.7 \pm 1.2$ & $<0.0001$ & $0.6 \pm 1.1$ & $1.5 \pm 0.8$ & 0.04 \\
\hline
\end{tabular}

${ }^{*}$ Controls versus iRBD patients; $t$ test for age; $\chi^{2}$ for sex, Mann-Whitney $U$ test for UPDRS and MoCA.

${ }^{\dagger}$ Mean $\pm S D$, with range in parentheses.

${ }^{\ddagger}$ In B controls, Mini-Mental State Examination (MMSE; maximum of 30 points) was used instead of MoCA.

MoCA = Montreal Cognitive Assessment; UPDRS-III = part 3 of the Unified Parkinson Disease Rating Scale (2003 version); NA = not available.

Values are median, with interquartile range in parentheses. 
TABLE 2

Demographic Data of PD Patients and Corresponding Controls

\begin{tabular}{lccc}
\hline \multicolumn{1}{c}{ Variable } & Controls & PD-NC & PD-MCl \\
\hline$n$ & 44 & 18 & 20 \\
Age & $68.8 \pm 8.7$ & $69.1 \pm 7.4$ & $73.8 \pm 5.7$ \\
Sex $(n$ male) & $32(73 \%)$ & $11(55 \%)$ & $14(70 \%)$ \\
RBD $(n)$ & & $8(44 \%)$ & $16(80 \%)$ \\
H\&Y stage $1(n)$ & & $14(78 \%)$ & 9.032 \\
H\&Y stage $2(n)$ & $4(22 \%)$ & $11(55 \%)$ \\
PD symptom duration (mo) & & $20.7 \pm 14.7$ & $16.9 \pm 12.9$ \\
UPDRS-III & $29.1 \pm 0.9$ & $12.9 \pm 6.1$ & $17.2 \pm 7.0$ \\
MMSE & $0 \pm 1$ & $28.7 \pm 1.0$ & $26.9 \pm 2.7$ \\
PDRP $z$ score & $0 \pm 1$ & $1.29 \pm 0.30$ & $2.60 \pm 1.56$ \\
iRBDRP $z$ score & & $0.98 \pm 1.1$ & $2.26 \pm 1.3$ \\
\hline
\end{tabular}

components that could best discriminate between controls and patients (26) were linearly combined to form 1 disease-related pattern (the iRBDRP). In this linear combination, each component was weighted by the coefficient resulting from the logistic regression model. All voxel weights in the iRBDRP were overlaid on a T1 MRI template in Montreal Neurologic Institute space for visualization.

\section{IRBDRP Subject Scores in Cohort B}

Anatomically registered images were masked and log-transformed, and subject and group means were removed to obtain a residual profile for each scan. The mask and group mean were based on cohort $\mathrm{A}$ in the iRBDRP identification process. The subject score was calculated by multiplying the residual profile of each subject with the pattern (12).

In cohort $\mathrm{B}, \mathrm{iRBDRP}$ subject scores in controls and iRBD patients were z-transformed to cohort A controls (i.e., the reference; $n=19$ ). iRBDRP $z$ scores were compared between cohort B controls and patients with a Student $t$ test. If significant, the iRBDRP was considered valid.

\section{RBDRP Subject Scores in De Novo PD Patients}

To account for differences in data acquisition, iRBDRP subject scores in the PD cohort were z-transformed to the subject scores of the corresponding 44 controls. For reference, we also calculated subject scores for the PDRP $(16,21)$ in the 44 controls and 38 PD patients. Again, PDRP subject scores were z-transformed with reference to the 44 controls (supplemental materials [available at http://jnm. snmjournals.org]).

\section{Stable Regions in iRBDRP}

Voxel weights in SSM/PCA patterns can fluctuate to some degree depending on the specific sample of patients and controls that is used for derivation (27). This is especially relevant in the study of iRBD because it is a heterogeneous patient group. To investigate which regions in the iRBDRP were stable, we performed a bootstrap resampling (1,000 repetitions). Voxels that survived a 1-sided confidence interval threshold of $90 \%$ (percentile method) after bootstrapping were overlaid on a T1 MRI template. The (stable) regions in the iRBDRP were compared visually to stable regions in the PDRP.

\section{Statistical Analysis}

iRBDRP $z$ scores were compared between cohort B controls and iRBD patients with an independent samples $t$ test. iRBDRP $z$ scores were also compared across controls, PD-RBD-, and PD-RBD+ groups with a 1-way ANOVA. Post hoc comparisons were Bonferronicorrected. This analysis was repeated for the comparisons of $\mathrm{H} \& \mathrm{Y}$ stage 1 versus $\mathrm{H} \& \mathrm{Y}$ stage 2, and PD-NC versus PD-MCI.

In the 44 controls and $38 \mathrm{PD}$ patients, the correlation between iRBDRP and PDRP subject $z$ scores was tested for significance with a Pearson $r$ correlation coefficient. In addition, a voxelwise correlation of the 2 patterns was performed with a Pearson $r$ correlation coefficient.

\section{RESULTS}

\section{iRBDRP Identification-Cohort A}

After the application of SSM/PCA to cohort A, the first 10 PCs (explaining $49.8 \%$ of the variance) were used for further analysis. The iRBDRP was formed by a linear combination of PC4 and 5 (5.5\% and $4.1 \%$ of variance, respectively), having approximately equal weights. All voxel weights in the iRBDRP contribute to the iRBDRP subject score (Fig. 1). Stable regions (90\% confidence interval threshold after bootstrap resampling) are shown in Figure 2 and include relative hypermetabolism in cerebellum, brain stem, thalamus, sensorimotor cortex, and left hippocampus/parahippocampal gyrus and relative hypometabolism in middle cingulate, temporal, occipital, and parietal cortices.

\section{iRBDRP Subject Scores in Cohort B iRBD Patients and Controls}

iRBDRP subject $z$ scores were significantly different between controls $(n=13)$ and iRBD patients $(n=9)$ from cohort B $(P=$ 0.04; Table 1; Fig. 3). iRBDRP subject scores in cohort B controls were not significantly different from subject scores in cohort A controls $(P=0.12)$. iRBDRP subject scores were also not significantly different between the 2 iRBD groups $(P=0.69)$.

\section{iRBDRP Subject Scores in PD Patients}

iRBDRP subject $z$ scores were significantly higher in PD patients than in controls $(P<0.0001)$. iRBD $z$ scores were higher 


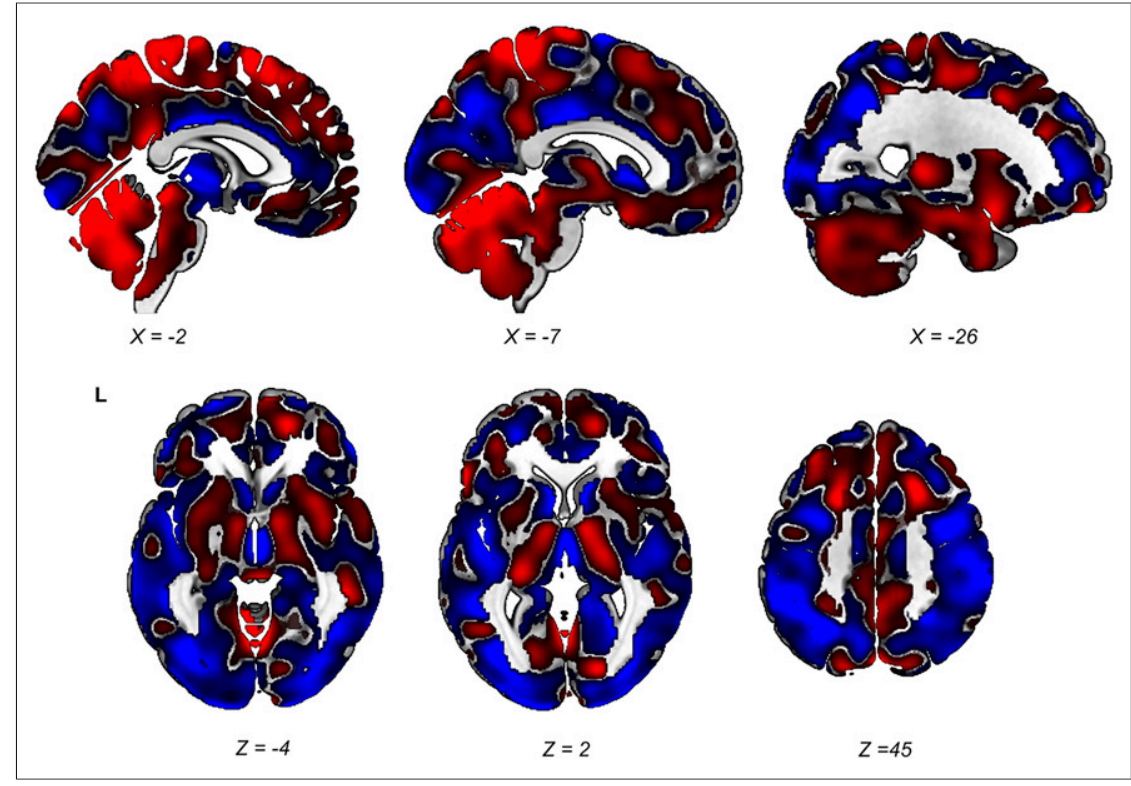

FIGURE 1. Unthresholded iRBDRP overlaid on T1 MRI template. Red indicates positive voxel weights (relative hypermetabolism), and blue indicates negative voxel weights (relative hypometabolism). $\mathrm{L}=$ left. Coordinates in axial $(\mathrm{Z})$ and sagittal $(\mathrm{X})$ planes are in Montreal Neurologic Institute standard space.

in patients with $\mathrm{H} \& \mathrm{Y}$ stage 1 than $\mathrm{H} \& \mathrm{Y}$ stage 2 (Fig. $4 \mathrm{~A}$ ), although this difference was not significant $(P=0.26)$. iRBD $z$ scores were also not significantly different between PD-RBDand PD-RBD+ (Fig. 4C). However, iRBDRP $z$ scores were significantly higher in PD-MCI patients than in PD-NC patients (Fig. 4B). Compared with the PD-NC group, the PD-MCI group was older and contained a larger proportion of patients with

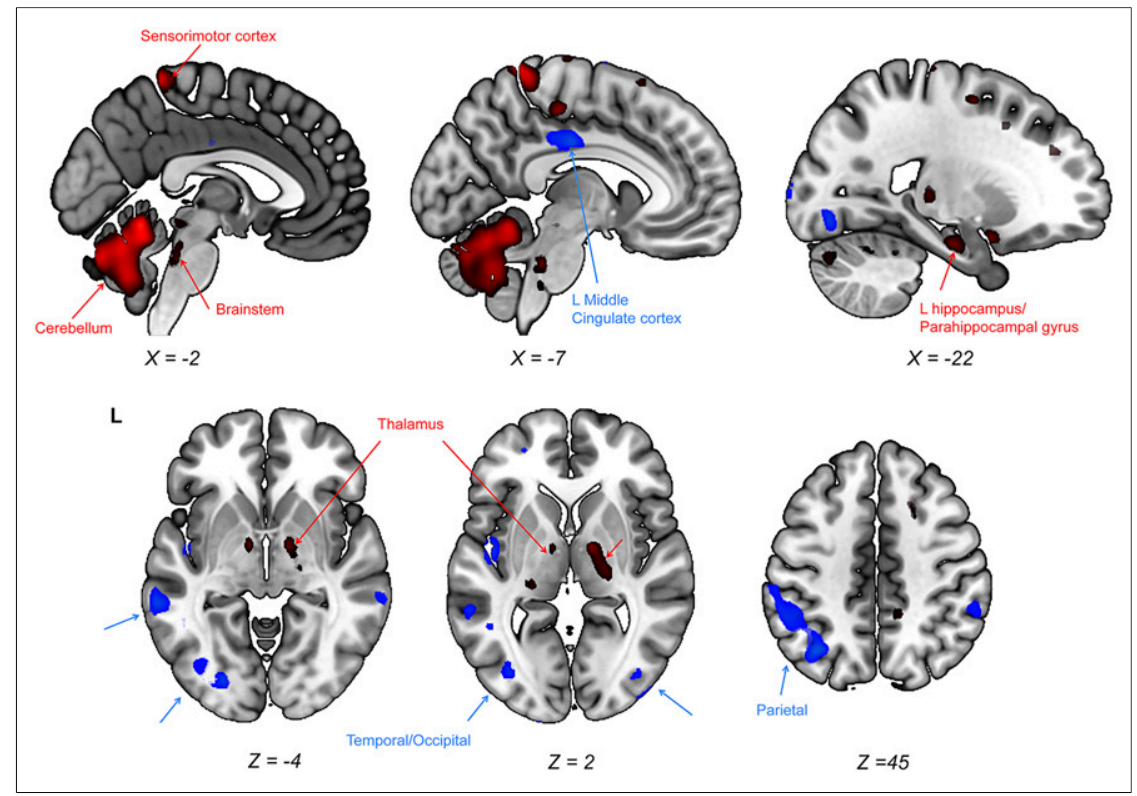

FIGURE 2. Stable voxels $(90 \%$ confidence interval not straddling zero after bootstrap resampling) of iRBDRP are visualized by overlaying them on T1 MRI template. Red indicates positive voxel weights (relative hypermetabolism), and blue indicates negative voxel weights (relative hypometabolism). $\mathrm{L}=$ left. Coordinates in axial $(\mathrm{Z})$ and sagittal $(\mathrm{X})$ planes are in Montreal Neurologic Institute standard space. concomitant probable RBD and a larger proportion of patients with bilateral parkinsonism (Table 2).

\section{Comparison with PDRP}

PDRP $z$ scores were also compared between the different PD subgroups and showed trends similar to the iRBDRP. Specifically, PDRP expression was not significantly different between PD-RBD - and $\mathrm{PD}-\mathrm{RBD}+(P=1.00)$, but was significantly different between $\mathrm{H} \& \mathrm{Y}$ stage 1 and H\&Y stage 2 PD patients $(P=0.024)$ and between PD-NC and PD-MCI $(P=0.004)$ (supplemental materials). Both PDRP and iRBDRP subject $z$ scores correlated significantly to age in PD patients $(r=0.50 ; P<$ $0.005)$ but not in controls $(r=0.30 ; P>$ 0.05). Both PDRP and iRBDRP subject scores were not significantly correlated to disease duration in PD patients $(\mathrm{r} \approx 0.02$, $P>0.80)$.

Subjects' iRBDRP and PDRP $z$ scores were highly correlated $(r=0.94 ; P<$ 0.0001 ; controls and PD patients combined). In addition, voxel weights of the PDRP were correlated to the iRBDRP $(r=0.52)$. For reference, voxel weights of 2 PDRPs from independent populations (the PDRP used in this study (16) vs. the original North-American PDRP published by Eidelberg et al. (14)) have a stronger voxelwise correlation $(r=0.75)$.

Stable regions (i.e., those surviving the $90 \%$ confidence interval threshold) in both the PDRP and the iRBDRP were overlaid on a T1 template. Figure 5A shows the relatively hypermetabolic stable regions of both patterns. Cerebellum, brain stem, thalamus, and sensorimotor cortex were hypermetabolic in both patterns. In contrast to the PDRP, putamen and pallidum did not show stable involvement in the iRBDRP. Stable hypometabolic regions (Fig. 5B) in the 2 patterns overlapped in parietal, temporal, and occipital cortices.

\section{DISCUSSION}

We report the second identification of the iRBDRP in an independent cohort of iRBD patients. The iRBDRP identified in this study disclosed a symmetric topography, which was strikingly similar to the PDRP. Both patterns are characterized by relatively increased metabolism in cerebellum, brain stem, and thalamus and by decreased metabolism in occipital, temporal, and parietal cortices. Furthermore, iRBDRP and PDRP subject scores were highly correlated, and both patterns were significantly expressed in de novo PD patients compared with controls.

In contrast to the original iRBDRP study by Wu et al. (20), we report slightly higher (nonsignificant) iRBDRP $z$ scores in patients 


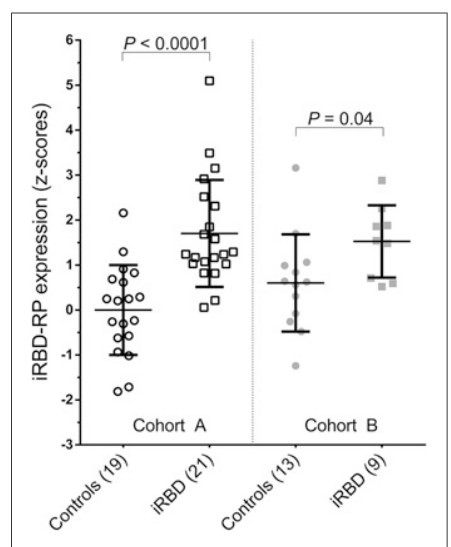

FIGURE 3. iRBDRP subject scores in derivation cohort (A) and in validation cohort (B). Subject scores were z-transformed to cohort A controls and compared between groups with a Student $t$ test. study. Considering that our iRBDRP and PDRP showed considerable overlap, subject scores on the 2 patterns were highly correlated, and that PDRP expression in $\mathrm{IRBD}$ is associated with a higher risk of conversion to PD (19), we hypothesize that the iRBDRP represents an early PDRP pattern. In other words, we suggest that the PDRP and iRBDRP are part of the same spectrum and are both likely to increase with disease progression.

Interestingly, iRBDRP expression was not significantly higher in PD patients with probable RBD than in PD patients without RBD, suggesting that the iRBDRP is not strictly related to the presence of RBD in PD. In addition, both PDRP and iRBDRP expression were higher in PD-MCI than PD-NC. The PD-MCI group was older and contained a larger proportion of patients with bilateral parkinsonism, and most had probable RBD. This combination of features may signal a rapidly progressive subtype of $\mathrm{PD}(22,28)$. The fact that such more severely affected PD patients have higher subject scores on both the PDRP and the iRBDRP again suggests that both patterns are markers of severity of the same disease process.

However, some important differences between the iRBDRP and the PDRP were found. The PDRP is characterized by relative hypermetabolism of putamen and pallidum. Although putamen and pallidum were relatively hypermetabolic in the unthresholded iRBDRP, they did not survive our predefined threshold (bootstrap resampling), which indicates that these regions were not involved in each iRBD patient. Relatively increased putaminal metabolism is thought to be a functional response to loss of dopaminergic input beyond a certain threshold and is related to the onset of motor symptoms (29). In a previous study, we showed that 9 of our 21 iRBD patients (cohort A) had significant loss of dopamine transporter binding (21), indicating neurodegeneration of the presynaptic dopaminergic system $(30,31)$. These 9 patients may have contributed to the relative hypermetabolism of putamen/pallidum in the unthresholded iRBDRP.

Furthermore, iRBDRP disclosed relative hypermetabolism of the dorsal aspect of the pons. Pontine hypermetabolism was more extensive in the PDRP. Nuclei that regulate REM sleep circuitry are located in the dorsal pons (32) and lie close to the noradrenergic locus coeruleus, cholinergic pedunculopontine nucleus, and serotonergic raphe nuclei. Although the spatial resolution of ${ }^{18}$ F-FDG PET images is not sufficient to discriminate between brain stem nuclei, we note that the clusters in the pons and mesencephalon (Fig. 2) overlap with the median raphe (33), locus coeruleus (34), and partially with the pedunculopontine nucleus $(35,36)$. These nuclei are affected early on in PD, before degeneration of the dopaminergic system (8). All 3 systems project to cerebellum and thalamus (3739). The pedunculopontine nucleus additionally projects to the basal
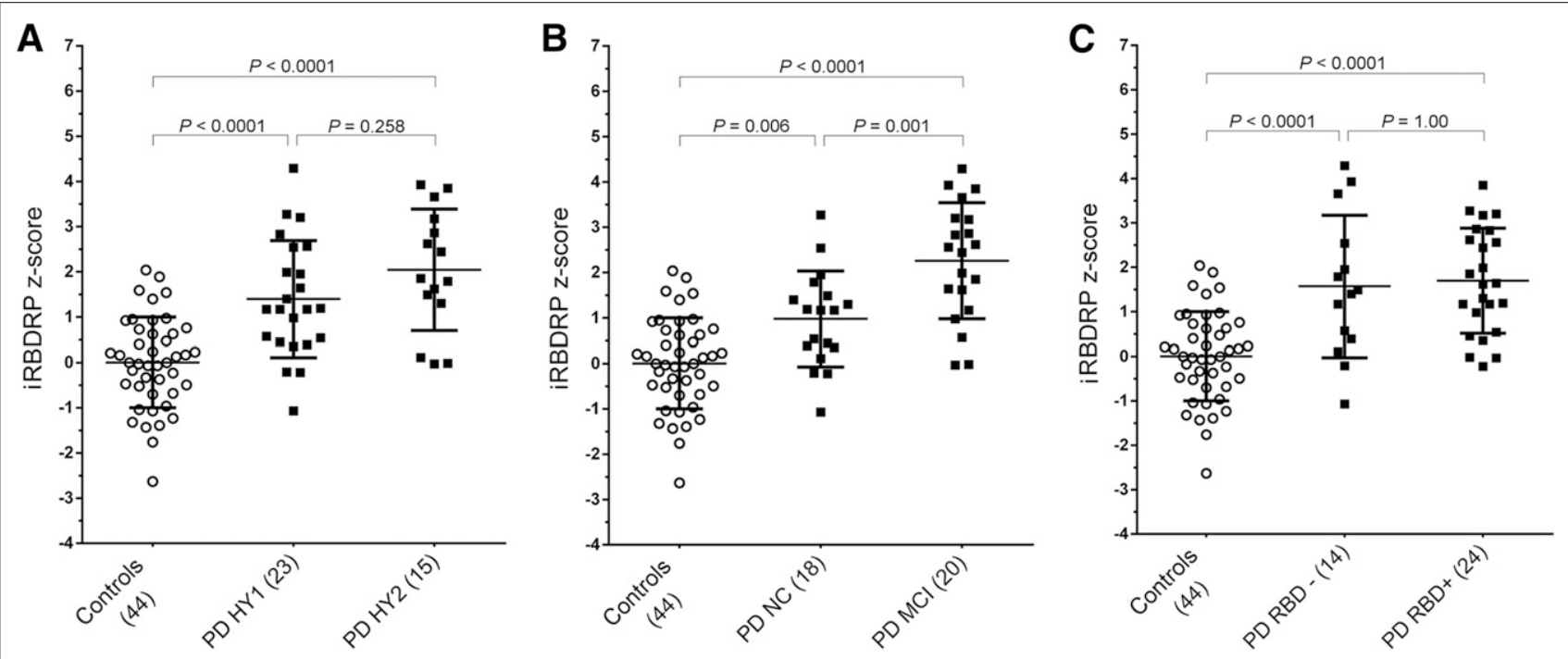

FIGURE 4. (A) IRBDRP subject scores were calculated in controls $(n=44)$, PD patients with H\&Y stage $1(n=23)$, and PD patients with H\&Y stage $2(n=15)$. Subject scores were $z$-transformed with reference to the 44 controls. IRBDRP $z$ scores were compared across groups with 1 -way ANOVA $\left(F_{(81)}=22.4, P<0.0001\right)$. IRBDRP $z$ scores are significantly higher in PD patients than in controls, but not significantly different between $\mathrm{H} \& Y$ stage 1 and H\&Y stage 2 groups. (B) IRBDRP $z$ scores were compared across controls, PD-NC, and PD-MCl with a 1 -way ANOVA $\left(F_{(81)}=30.2, P<0.0001\right)$. (C) IRBDRP $z$ scores were compared across controls, PD-RBD- $(n=14)$, and PD-RBD $+(n=24)$ with a 1-way ANOVA $\left(F_{(81)}=20.3, P<0.0001\right)$. $P$ values in post hoc group comparisons were Bonferroni-corrected. 


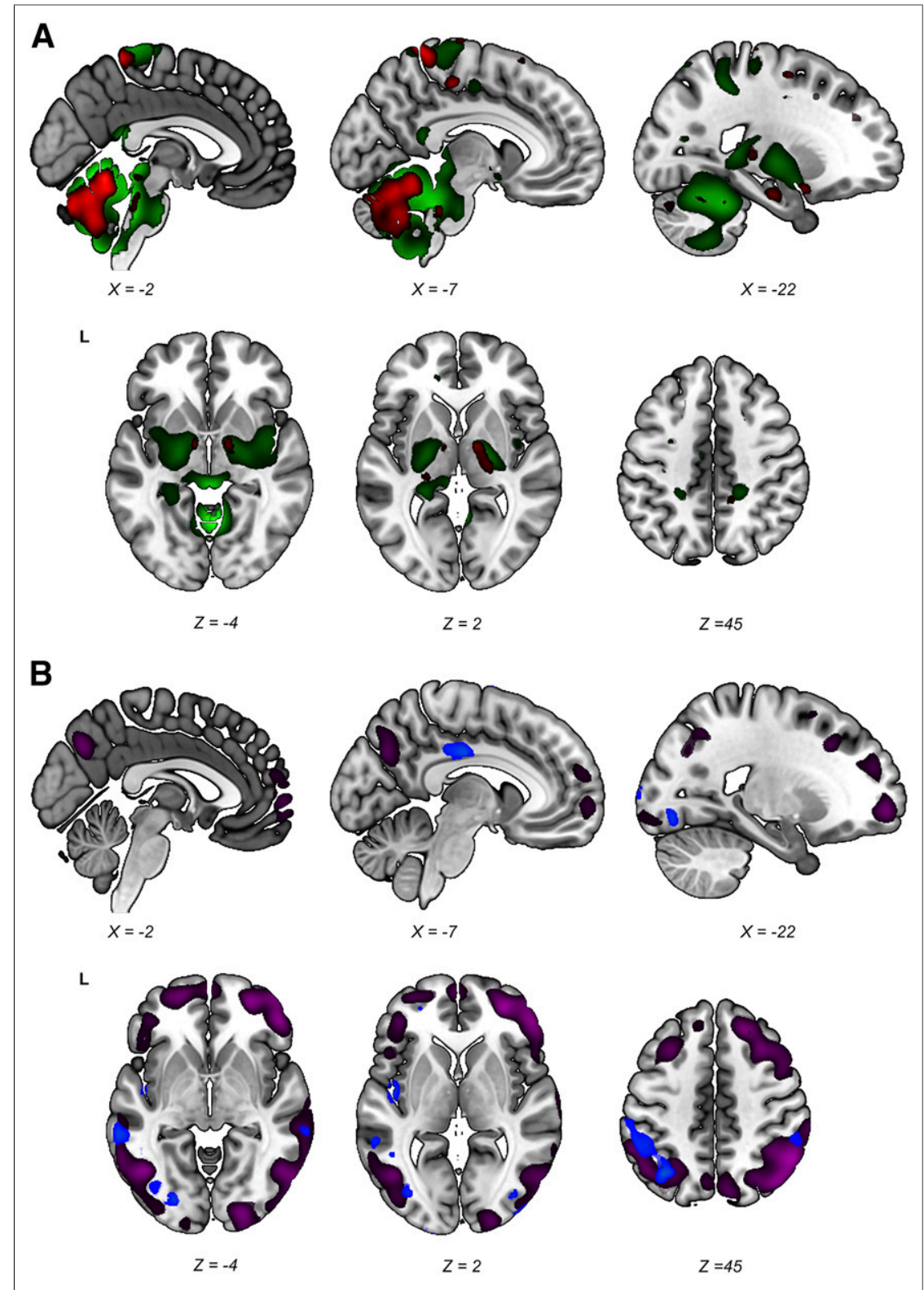

FIGURE 5. Stable regions in iRBDRP and PDRP overlap. Stable voxels $(90 \%$ confidence interval not straddling zero after bootstrap resampling) of iRBDRP and PDRP are overlaid on T1 MRI template. (A) Stable, relatively hypermetabolic regions of PDRP (green) and iRBDRP (red). (B) Stable, relatively hypometabolic regions of PDRP (purple) and iRBDRP (blue). $\mathrm{L}=$ left. Coordinates in axial $(\mathrm{Z})$ and sagittal $(X)$ planes are in Montreal Neurologic Institute standard space.
(44-47), appears to be a distinct feature of the iRBDRP, as it was not seen in the PDRP.

Both the original (20) and the current iRBDRP included relative hypermetabolism of the thalamus, hippocampus, and pons and relative hypometabolism of the temporal and occipital cortices. However, there are clear differences. First, in contrast to the original iRBDRP, our pattern included relative hypermetabolism of the cerebellum, putamen, and pallidum, in keeping with Holtbernd et al. (19). The 2 latter regions were not considered stable in our analysis, but did contribute to iRBDRP subject scores. Second, Wu et al. described relatively increased metabolism of the middle cingulate, whereas our iRBDRP discloses relatively decreased metabolism of the same region. Third, relative hypometabolism of the parietal cortex appears to be a more salient feature in the current iRBDRP, whereas the occipital cortex was more prominent in the original iRBDRP.

It is conceivable that the differences between the 2 iRBDRPs were caused by heterogeneity in the respective iRBD samples. Although the iRBD cohorts in both studies had similar ages and symptom durations, it is unknown which proportion of patients will develop DLB or PD, and at what time interval. For example, it is possible that a larger proportion of prodromal DLB patients in the study by Wu et al. has caused the salient reductions in the occipital cortex. In addition, iRBD patients occasionally develop multiple system atrophy instead of PD or DLB (1-6). Multiple system atrophy is characterized by a different metabolic pattern (17) and could therefore have influenced pattern topography and subject scores.

Furthermore, the iRBDRP in our study was formed by a combination of PCs 4 and 5 (together accounting for $9.6 \%$ of the total variance), whereas most diseaserelated patterns (such as the PDRP) are ganglia and motor cortex (37), the locus coeruleus projects to the hippocampus and cortex (38), and the median raphe projects to the hippocampus and cingulate (39). All these regions were identified in the iRBDRP and PDRP. Although the underlying mechanism of relative pontine hyperactivity is unclear, it appears to be a consistent feature of iRBD $(19,20,40)$ and PD $(11-18)$.

Relative hippocampal hypermetabolism, another consistent finding in functional imaging studies in iRBD $(20,41-43)$, reliably contributed to the iRBDRP, but not to the PDRP. Relative hyperperfusion of the hippocampus was associated with subsequen development of PD/DLB $(n=10)$ in a 3 -y clinical follow-up study of 20 iRBD patients (40). Hypometabolism of the middle cingulate, associated with cognitive decline in longitudinal PD studies found among the first few PCs (i.e., PC 1 and PC 2 combined (16), or PC 1 in isolation $(14,18)$ ). Wu et al. also identified their iRBDRP by PC 1 (14\% of the variance) (20). The fact that the current iRBDRP was found among components with lower eigenvalues indicates that the between-subject variance was larger than the between-group variance in the iRBDRP identification cohort (cohort A: iRBD vs. controls). We also evaluated components 1 and 2 in cohort $A$. These components did not discriminate significantly between controls and iRBD patients in cohort A and cohort B, and were also not significantly different between PD patients and healthy controls $(P>0.05)$. PC 1 and PC 2 were similar to the first 2 components, which were reported in several cohorts of healthy controls (48). This 
is perhaps not surprising, as we contrasted controls to patients who did not have parkinsonism, of which most $(n=12)$ had normal dopamine transporter scans. The disease-related alterations are weak; a proportion of patients may have a metabolic brain profile that is close to normal. As a consequence, the first few PCs in this dataset describe normal resting-state brain function.

\section{CONCLUSION}

Our results suggest that the iRBDRP is an early manifestation of the PDRP. Expression of both PDRP and iRBDRP was higher in patients with a more severe form of PD (PD-MCI), which may indicate that expression of the 2 patterns increases with disease severity. This finding may be relevant for future progression and therapeutic studies in prodromal PD. Clinical and imaging followup of our cohort is ongoing and will provide insights to the changes of the iRBDRP over time and in relation to phenoconversion to PD or DLB.

\section{DISCLOSURE}

This study was funded by the Dutch 'Stichting ParkinsonFonds' and the German 'ParkinsonFonds Deutschland'. Wolfgang H. Oertel, MD, PhD is Hertie-Senior-Research Professor, supported by the Charitable Hertie Foundation, Frankfurt/Main, Germany. No other potential conflict of interest relevant to this article was reported.

\section{ACKNOWLEDGMENTS}

Members of the REMPET Study Group include Elisabeth SittigWiegand, Candan Depboylu, Kathrin Reetz, Sebastiaan Overeem, Angelique Pijpers, Fransje E. Reesink, Teus van Laar, Laura K. Teune, Helmut Höffken, Marcus Luster, Lars Timmermann, Karl Kesper, Sofie M. Adriaanse, Jan Booij, Gianmario Sambuceti, Nicola Girtler, and Cathrine Jonsson. We thank J.M.C. (Marc) van Dijk, MD, PhD, and D.L.M. (Marinus) Oterdoom, MD, from the neurosurgery department, UMCG, for assistance in localizing brainstem regions in the iRBDRP. We thank Rosalie V. Kogan for proofreading the manuscript.

\section{REFERENCES}

1. Postuma RB, Gagnon JF, Vendette M, Fantini ML, Massicotte-Marquez J, Montplaisir J. Quantifying the risk of neurodegenerative disease in idiopathic REM sleep behavior disorder. Neurology. 2009;72:1296-1300.

2. Postuma RB, Lang AE, Gagnon JF, Pelletier A, Montplaisir JY. How does parkinsonism start? Prodromal parkinsonism motor changes in idiopathic REM sleep behaviour disorder. Brain. 2012;135:1860-1870.

3. Iranzo A, Tolosa E, Gelpi E, et al. Neurodegenerative disease status and post-mortem pathology in idiopathic rapid-eye-movement sleep behaviour disorder: an observational cohort study. Lancet Neurol. 2013;12:443453.

4. Schenck CH, Boeve BF, Mahowald MW. Delayed emergence of a parkinsonian disorder or dementia in $81 \%$ of older men initially diagnosed with idiopathic rapid eye movement sleep behavior disorder: a 16-year update on a previously reported series. Sleep Med. 2013;14:744-748.

5. Iranzo A, Fernandez-Arcos A, Tolosa E, et al. Neurodegenerative disorder risk in idiopathic REM sleep behavior disorder: study in 174 patients. PLoS One. 2014; 9:e89741.

6. Postuma RB, Iranzo A, Hogl B, et al. Risk factors for neurodegeneration in idiopathic rapid eye movement sleep behavior disorder: a multicenter study. Ann Neurol. 2015;77:830-839.
7. Boeve BF. Idiopathic REM sleep behaviour disorder in the development of Parkinson's disease. Lancet Neurol. 2013;12:469-482.

8. Braak H, Del Tredici K, Rub U, de Vos RA, Jansen Steur EN, Braak E. Staging of brain pathology related to sporadic parkinson's disease. Neurobiol Aging. 2003; 24:197-211.

9. Berg D, Postuma RB, Adler CH, et al. MDS research criteria for prodromal Parkinson's disease. Mov Disord. 2015;30:1600-1611.

10. Postuma RB, Berg D, Stern M, et al. MDS clinical diagnostic criteria for Parkinson's disease. Mov Disord. 2015;30:1591-1601.

11. Eidelberg D. Metabolic brain networks in neurodegenerative disorders: a functional imaging approach. Trends Neurosci. 2009;32:548-557.

12. Spetsieris PG, Eidelberg D. Scaled subprofile modeling of resting state imaging data in parkinson's disease: methodological issues. Neuroimage. 2011;54:28992914.

13. Eidelberg D, Moeller JR, Dhawan V, et al. The metabolic topography of parkinsonism. J Cereb Blood Flow Metab. 1994;14:783-801.

14. Ma Y, Tang C, Spetsieris PG, Dhawan V, Eidelberg D. Abnormal metabolic network activity in Parkinson's disease: test-retest reproducibility. J Cereb Blood Flow Metab. 2007;27:597-605.

15. Niethammer M, Eidelberg D. Metabolic brain networks in translational neurology: concepts and applications. Ann Neurol. 2012;72:635-647.

16. Teune LK, Renken RJ, de Jong BM, et al. Parkinson's disease-related perfusion and glucose metabolic brain patterns identified with PCASL-MRI and FDG-PET imaging. Neuroimage Clin. 2014;5:240-244.

17. Teune LK, Renken RJ, Mudali D, et al. Validation of parkinsonian diseaserelated metabolic brain patterns. Mov Disord. 2013;28:547-551.

18. Wu P, Wang J, Peng S, et al. Metabolic brain network in the Chinese patients with Parkinson's disease based on ${ }^{18}$ F-FDG PET imaging. Parkinsonism Relat Disord. 2013;19:622-627.

19. Holtbernd F, Gagnon JF, Postuma RB, et al. Abnormal metabolic network activity in REM sleep behavior disorder. Neurology. 2014;82:620627.

20. Wu P, Yu H, Peng S, et al. Consistent abnormalities in metabolic network activity in idiopathic rapid eye movement sleep behaviour disorder. Brain. 2014;137: 3122-3128.

21. Meles SK, Vadasz D, Renken RJ, et al. FDG PET, dopamine transporter SPECT, and olfaction: combining biomarkers in REM sleep behavior disorder. Mov Disord. 2017;32:1482-1486.

22. Fereshtehnejad SM, Postuma RB. Subtypes of parkinson's disease: what do they tell us about disease progression? Curr Neurol Neurosci Rep. 2017; $17: 34$.

23. Arnaldi D, Morbelli S, Brugnolo A, et al. Functional neuroimaging and clinical features of drug naive patients with de novo Parkinson's disease and probable RBD. Parkinsonism Relat Disord. 2016;29:47-53.

24. Boeve BF, Molano JR, Ferman TJ, et al. Validation of the mayo sleep questionnaire to screen for REM sleep behavior disorder in an aging and dementia cohort. Sleep Med. 2011;12:445-453.

25. Della Rosa PA, Cerami C, Gallivanone F, et al. A standardized $\left[{ }^{18} \mathrm{~F}\right]$-FDG-PET template for spatial normalization in statistical parametric mapping of dementia. Neuroinformatics. 2014;12:575-593.

26. Akaike H. A new look at the statistical model identification. IEEE Trans Automat Contr. 1974;19:716-723.

27. Habeck C, Foster NL, Perneczky R, et al. Multivariate and univariate neuroimaging biomarkers of Alzheimer's disease. Neuroimage. 2008;40:15031515

28. Zhu RL, Xie CJ, Hu PP, Wang K. Clinical variations in Parkinson's disease patients with or without REM sleep behaviour disorder: a meta-analysis. Sci Rep. 2017;7:40779-40787.

29. Tang CC, Poston KL, Dhawan V, Eidelberg D. Abnormalities in metabolic network activity precede the onset of motor symptoms in Parkinson's disease. J Neurosci. 2010;30:1049-1056.

30. Iranzo A, Lomena F, Stockner H, et al. Decreased striatal dopamine transporter uptake and substantia nigra hyperechogenicity as risk markers of synucleinopathy in patients with idiopathic rapid-eye-movement sleep behaviour disorder: a prospective study [corrected]. Lancet Neurol. 2010;9:1070-1077.

31. Iranzo A, Valldeoriola F, Lomena F, et al. Serial dopamine transporter imaging of nigrostriatal function in patients with idiopathic rapid-eye-movement sleep behaviour disorder: a prospective study. Lancet Neurol. 2011;10:797805.

32. Peever J, Luppi PH, Montplaisir J. Breakdown in REM sleep circuitry underlies REM sleep behavior disorder. Trends Neurosci. 2014;37:279-288.

33. Kranz GS, Hahn A, Savli M, Lanzenberger R. Challenges in the differentiation of midbrain raphe nuclei in neuroimaging research. Proc Natl Acad Sci USA. 2012;109:E2000. 
34. Keren NI, Lozar CT, Harris KC, Morgan PS, Eckert MA. In vivo mapping of the human locus coeruleus. Neuroimage. 2009;47:1261-1267.

35. Zrinzo L, Zrinzo LV, Tisch S, et al. Stereotactic localization of the human pedunculopontine nucleus: atlas-based coordinates and validation of a magnetic resonance imaging protocol for direct localization. Brain. 2008;131:1588-1598.

36. Janzen J, van 't Ent D, Lemstra AW, Berendse HW, Barkhof F, Foncke EM. The pedunculopontine nucleus is related to visual hallucinations in Parkinson's disease: preliminary results of a voxel-based morphometry study. J Neurol. 2012;259: $147-154$.

37. Benarroch EE. Pedunculopontine nucleus: Functional organization and clinical implications. Neurology. 2013;80:1148-1155.

38. Delaville C, Deurwaerdere PD, Benazzouz A. Noradrenaline and Parkinson's disease. Front Syst Neurosci. 2011;5:31-43.

39. Huot $\mathrm{P}$, Fox SH. The serotonergic system in motor and non-motor manifestations of Parkinson's disease. Exp Brain Res. 2013;230:463-476.

40. Dang-Vu TT, Gagnon JF, Vendette M, Soucy JP, Postuma RB, Montplaisir J. Hippocampal perfusion predicts impending neurodegeneration in REM sleep behavior disorder. Neurology. 2012;79:2302-2306.

41. Mazza S, Soucy JP, Gravel P, et al. Assessing whole brain perfusion changes in patients with REM sleep behavior disorder. Neurology. 2006;67:1618-1622.
42. Vendette M, Gagnon JF, Soucy JP, et al. Brain perfusion and markers of neurodegeneration in rapid eye movement sleep behavior disorder. Mov Disord. 2011;26:1717-1724.

43. Ge J, Wu P, Peng S, et al. Assessing cerebral glucose metabolism in patients with idiopathic rapid eye movement sleep behavior disorder. J Cereb Blood Flow Metab. 2015;35:2062-2069.

44. Bohnen NI, Koeppe RA, Minoshima S, et al. Cerebral glucose metabolic features of Parkinson disease and incident dementia: longitudinal study. J Nucl Med. 2011;52:848-855.

45. Tard C, Demailly F, Delval A, et al. Hypometabolism in posterior and temporal areas of the brain is associated with cognitive decline in Parkinson's disease. J Parkinsons Dis. 2015;5:569-574.

46. Pappatà S, Santangelo G, Aarsland D, et al. Mild cognitive impairment in drugnaive patients with PD is associated with cerebral hypometabolism. Neurology. 2011;77:1357-1362.

47. Garcia-Garcia D, Clavero P, Gasca Salas C, et al. Posterior parietooccipital hypometabolism may differentiate mild cognitive impairment from dementia in Parkinson's disease. Eur J Nucl Med Mol Imaging. 2012;39:1767-1777.

48. Spetsieris PG, Ko JH, Tang CC, et al. Metabolic resting-state brain networks in health and disease. Proc Natl Acad Sci USA. 2015;112:2563-2568. 